\title{
Theory and Practice of
} Logic Programming
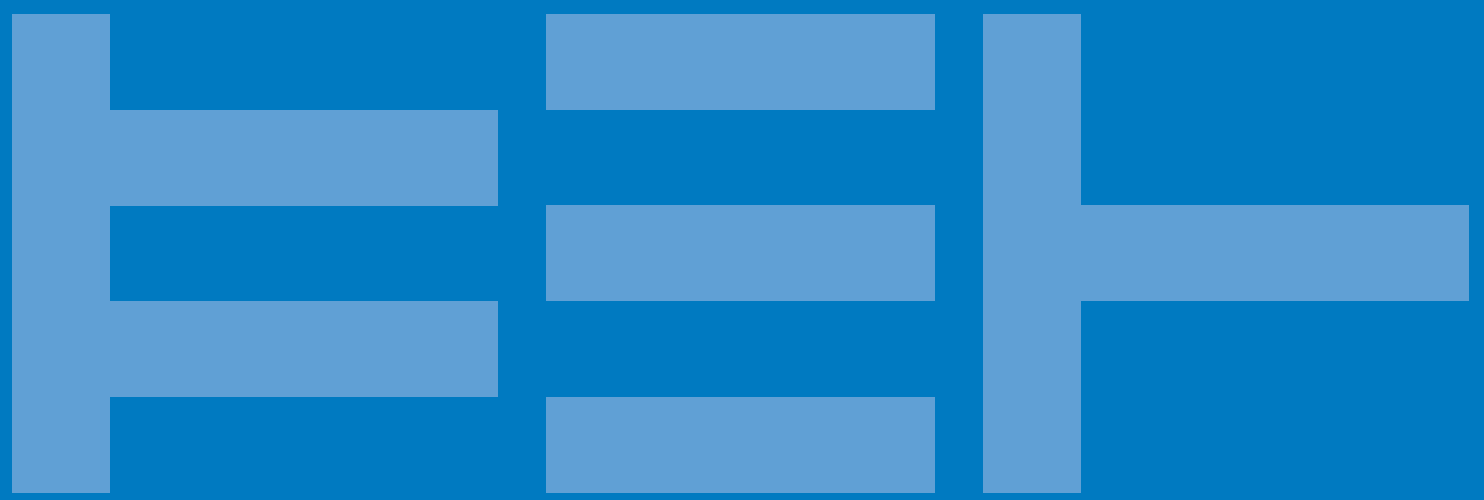

VOLUME 11 PARTS 4-5 JULY 2011

Special issue on

27 th International

Conference on Logic Programming

edited by John Gallagher and Michael Gelfond 
EDITOR-IN-CHIEF

Ilkka Niemelä

Aalto University School of Science and Technology

Department of Information and Computer Science

P.O. Box 15400, FI-00076 AALTO, FINLAND

EMAIL Ilkka.Niemela@tkk.fi
FOUNDING EDITOR-IN-CHIEF

Jack Minker

Department of Computer Science

and Institute for Advanced Computer Studies,

University of Maryland,

College Park, Maryland 20742, USA,

EMAIL minker@cs.umd.edu

\section{AREA EDITORS}

Constraints

Peter J. Stuckey

University of Melbourne

pjs@cs.mu.oz.au

Databases and Semantic Web Reasoning

Thomas Eiter

Vienna University of Technology

eiter@kr.tuwien.ac.at

Design, Analysis and Implementation of

Languages

Maria Garcia de la Banda

Monash University

Maria.GarciadelaBanda@infotech.

monash.edu.au

Book Reviews

Krzysztof Apt

CWI, The Netherlands

apt@cwi.nl
Inductive Logic Programming and

Multi-relational Data Mining

Luc De Raedt

Katholieke Universiteit Leuven

luc.deraedt@cs.kuleuven.be

Knowledge Representation and

Nonmonotonic Reasoning

Michael Gelfond

Texas Tech. University

Michael.Gelfond@ttu.edu

Logic Programming Methodology \& Applications

Gopal Gupta

The University of Texas at Dallas

gupta@utdallas.edu
Specification, Analysis and Verification of Systems

Michael Leuschel

University of Düsseldorf

leuschel@cs.uni-dusseldorf.de

Theory

Giorgio Levi

Universitá di Pisa

levi@di.unipi.it

Technical Notes

Catuscia Palamidessi

INRIA LIX

catuscia@lix.polytechnique.fr

EDITORIAL ADVISORS

\section{Baral}

Arizona State University

A. Bossi

Università Ca'Foscari

di Venezia

Maurice Bruynooghe

Katholieke Universiteit

Leuven

M. Carlsson

Swedish Institute of

Computer Science

A. Colmerauer

Université d'Aix Marseille II

V. Dahl

Simon Fraser University

D. De Schreye

Katholieke Universiteit

Leuven

J. Dix

Clausthal University of

Technology, Germany

P.M. Dung

Asian Institute of

Technology, Thailand
P. Flach

University of Bristol

M. Gabbrielli

University of Bologna

J. Gallagher

Roskilde University

Manuel Hermenegildo

Universidad Politecnica

de Madrid

K. Inoue

National Institute of

Informatics, Japan

Joxan Jaffar

National University of

Singapore

A. Kakas

University of Cyprus

A. King

University of Kent

R.A. Kowalski

Imperial College

L. Libkin

University of Toronto
V. Lifschitz

University of Texas at

Austin

M. Maher

National ICT Australia

D. Miller

INRIA, École

Polytechnique

S. Muggleton

Imperial College

G. Nadathur

University of Minnesota

R. O'Keefe

University of Otago, New

Zealand

L.M. Pereira

Universidade Nova de

Lisboa

J.A. Robinson

Highland Institute

F. Rossi

Università di Padova

K. Sagonas

Uppsala University

\section{INTERNET ACCESS}

The journal is included in the Cambridge Journals Online service which can be found at journals.cambridge.org/. Refer also for other information to the dedicated journal web site at http://logicprogramming.org/ 